\title{
Eficacia antimicótica del aloe vera y la sangre de grado en comparación al Gynocanesten ${ }^{\circledR}$
}

\section{Performance of aloe vera antifungal dragon's blood and Compared to Gynocanesten}

\author{
César Cayo ${ }^{*}$, Cristian Escurra ${ }^{* *}$, Gleiky Robles ${ }^{* * *}$
}

http://dx.doi.org/10.21503/CienciayDesarrollo.2012.v15i2.02

\section{RESUMEN}

El presente trabajo se ha realizado con la finalidad de estudiar dos plantas naturales, como son la sangre de grado (Croton lechleri) y el Aloe vera (Aloe barbadensismiller) y determinar su eficacia como agentes antimicóticos en el tratamiento de las patologías vaginales, realizando un estudio comparativo con un producto control: el Gynocanesten. El grupo experimental evaluado estuvo constituido por 27 alumnas del IV ciclo de la E.A.P. de Obstetricia de la Facultad de Medicina Humana y Ciencias de la Salud, matriculadas en el curso de Embriología y Genética en el ciclo académico 2011-II. Las participantes se agruparon al azar, de la siguiente manera: Grupo No 01 (utilizó el producto control: Gynocanesten"), Grupo No 02 (utilizó el aloe vera) y el Grupo № 03 (utilizó la sangre de grado). El método empleado en el estudio es comparativo, prospectivo, longitudinal y cualitativo. De acuerdo con los resultados obtenidos de manera experimental, se evaluó durante 3 fechas la evolución de la infección vaginal con respecto al tratamiento natural aplicado, concluyendo que los productos empleados, el aloe vera y la sangre de grado, fueron tan eficaces como la aplicación del producto control Gynocanesten.

Palabras clave: aloe vera, sangre de grado, Gynocanesten ${ }^{\circ}$, flora micótica, tracto vaginal.

\section{ABSTRAC}

This work has been performed for the purpose of study two natural plants such as sangre de grado (Croton lechleri) and Aloe vera (aloe barbadensis miller), and from them determine their efficacy as antifungal agents in the treatment of pathologies vaginal, making a comparative study with a control product: the Gynocanesten . The experimental group evaluated consisted of 27 students of the fourth cycle of EAP Obstetrics, Faculty of Human Medicine and Health Sciences, enrolled in the course of Embryology and Genetics in the academic year 2001-II. Participants were grouped randomly as follows: Group No. 01 (used the product control: Gynocanesten"), Group No. 02 (used aloe vera) and Group No. 03 (used the blood of a degree). The method used in the study is comparative, prospective, longitudinal and qualitative. According to the results obtained experimentally evaluated for three dates the development of vaginal infection with respect to natural treatment applied, concluding that the products used, aloe vera and dragon's blood, were as effective as the application of the product Gynocanesten control.

Key words: aloe vera, sangre de grado, Gynocanesten', flora fungal, vaginal tract.

\footnotetext{
* Docente de Embriología y Genética de la EAP de Obstetricia de la Facultad de Medicina Humana y Ciencias de la Salud de la UAP, sede Huacho. E-mail: cesarcayorojas@hotmail.com

** Docente de Estadística de la UAP, sede Huacho. E-mail: crivan16@hotmail.com

*** Alumna del VII ciclo de la EAP de Obstetricia de la Facultad de Medicina Humana y Ciencias de la Salud de la UAP, sede Huacho. E-mail: glei_blexez@hotmail.com
} 


\section{INTRODUCCIÓN}

Dentro de los antecedentes relacionados con el trabajo de investigación, se tomaron en consideración los trabajos que se vienen realizando durante los últimos 15 años en diferentes entidades estatales de salud y del sector privado en nuestro país.

En el año 2001, Méndez M. y Calderón J. realizaron un estudio para evaluar la prevalencia de vaginosis bacteriana en la población de un centro de salud predominantemente rural de Quilmaná (Cañete), donde se obtuvo que la vaginosis bacteriana cumplía en el $33 \%$ de pacientes por lo menos con tres de los cuatro criterios de Amsel para el diagnóstico respectivo: candidiasis en el $15 \%$, tricomoniasis en el $4 \%$ y mixto en el $5 \%$. (4)

En países desarrollados, se encuentra vaginosis bacteriana en el $5 \%$ de las mujeres asintomáticas y en el $25 \%$ de las mujeres con síntomas ginecológi$\cos (26)$, y se ha visto que es más baja ( $4 \%)$ en estudiantes universitarias asintomáticas, y máxima (64\%) en pacientes de clínicas de enfermedades de transmisión sexual (1).

En estudios realizados a nivel mundial, se ha encontrado que el $90 \%$ de esas infecciones con causadas por tres grupos de agentes etiológicos: 1. Un grupo de bacterias anaerobias que producen vaginosis, 2. Levaduras del genero Candidaspp, y 3. Infecciones por parásito protozoario Trichomonasvaginalis. (6)

De estas, la vaginosis bacteriana es la más frecuente, y tiene como agentes causales bacterias que sustituyen la flora normal de Lactobacillus acidophilus, tales como Mobiluncus spp, Prevotella spp, Mycoplasma hominis o Gardnerella vaginalis. Esta última es la de mayor frecuencia entre los agentes bacterianos (3).

Además, se presentan investigaciones relacionadas con la acción bactericida y antifúngica, como reportó Bland (1985) en el sentido de que beber gel de aloe vera mejora la ingestión de las proteínas, ayuda a normalizar el movimiento intestinal, controla las infecciones de hongos, y mejora el síndrome del colon irritable, la acidez estomacal y la colitis (2).

Ortiz, Capcha, Palomino y Aguilar (2003) refieren la actividad antibacteriana de la sangre de grado frente a la bacteria. Los resultados obtenidos muestran que la actividad cicatrizante de la sangre de grado, probada en estudios anteriores y complementada con la actividad antibacteriana determinada en el presente estudio, sería la responsable de la capacidad curativa de este producto frente a las úlceras gástricas (5).

Por lo tanto, la importancia de nuestro trabajo de campo radica en que las mujeres puedan tratar y controlar de forma económica la proliferación anormal de colonias bacterianas y micóticas que producen las patologías infecciosas más comunes en nuestro país, y que generalmente son tratadas con fármacos de vía tópica, pudiendo estos tratamientos sobrellevarse de manera natural con medicina alternativa de largo plazo.

Por estas razones, nos planteamos el siguiente problema: ¿existe eficacia antimicótica del aloe vera y la sangre de grado en comparación con el Gynocanesten?

La hipótesis principal planteada es: sí existe eficacia antimicótica vaginal de la sangre de grado y el aloe vera, similar al producto control Gynocanesten. Las hipótesis derivadas son tres: 1) la sangre de grado como antimicótico vaginal es tan eficaz como el Gynocanesten', 2) el aloe vera como antimicótico vaginal es tan eficaz como el Gynocanesten', y 3) los tres productos aplicados son eficaces como antimicóticos vaginales.

Como objetivo principal se planteó el siguiente: determinar la eficacia antimicótica vaginal del 
aloe vera y la sangre de grado en comparación con el Gynocanesten, a través de tres objetivos específicos: 1) determinar la eficacia antimicótica vaginal entre la sangre de grado y el Gynocanesten ${ }^{\circ}$ 2) determinar la eficacia antimicótica vaginal entre el aloe vera y el Gynocanesten', y 3) determinar la interrelación que existe entre la aplicación de los tres productos y su eficacia antimicótica vaginal.

La elección de la muestra fue tomada de las alumnas del IV ciclo de la Escuela Académico-Profesional de Obstetricia, quedando de la siguiente manera: Grupo No 01 (emplearon el producto control: Gynocanesten ${ }^{\circ}$ ), Grupo $N^{\circ} 02$ (emplearon el aloe vera) y Grupo No 03 (emplearon la sangre de grado).

Seguidamente, para la realización del trabajo de campo, se llevó a cabo un muestreo al azar no probabilístico, a fin de elegir los grupos integrados por nueve personas en cada uno de los grupos ya mencionados.

Una limitación fue la escasa existencia de pruebas de trabajos de investigación relacionados con el nivel de eficacia de productos naturales como sangre de grado y aloe vera.

Finalmente, la oportunidad de lograr un avance significativo en el desarrollo de prevención de salud cobra mayor importancia, debiendo para ello vencer los grandes obstáculos que durante años han entorpecido el logro de una situación saludable del individuo, contándose entre estos las prácticas de higiene, los bajos ingresos económicos y la incidencia elevada de ITS. Es evidente, pues, la necesidad de optimizar la reducción de estas patologías con un tratamiento natural, económico y eficaz.

\section{MATERIALES Y MÉTODOS}

El estudio se desarrolló en la ciudad de Huacho durante el mes de mayo del 2011; la población es- tuvo conformada por las alumnas del IV ciclo de estudios de la Escuela Académico-Profesional de Obstetricia, de la Facultad de Medicina Humana y Ciencias de la Salud, matriculadas en el ciclo 2011-II, en la asignatura de Embriología y Genética. La muestra específica fue de 27 alumnas que asistían regularmente, con una edad promedio de 20 a 25 años. Para la elección de la muestra, se utilizó un diseño al azar no probabilístico que contempló la formación de tres grupos conformados por 9 alumnas. El Grupo No 1 utilizó el producto control: Gynocanesten', el Grupo No 02 empleó la sábila (Aloe vera), y el Grupo № 03 usó la sangre de grado (Croton lechleri).

En el manejo del experimento se consideraron cuatro variables de estudio: tres variables independientes, constituidas por dos productos naturales, la sábila (Aloe vera) y la sangre de grado (Croton lechleri), y el producto control Gynocanesten; y la variable dependiente, los niveles de flora micótica en el tracto vaginal, que fueron operaciones alisadas en cuatro niveles para cada uno.

Luego se procedió a realizar exámenes ginecológicos microbiológicos previos a la primera prueba de tratamiento con los productos mencionados. Los productos naturales fueron preparados previamente, de tal manera que la sábila sufrió un tratamiento de desyodificación durante 24 horas, y se procedió a aplicar. Con respecto a la sangre de grado, se aplicó en forma de tintura. Se procedió a realizar este proceso diariamente después de la higiene genital, vía tópica, con ayuda de los dispensadores que se adquirieron, tal como se hizo en el grupo control para optimizar la profundidad de los productos a nivel intravaginal. La aplicación del Gynocanesten fue vía 6 tabletas de $100 \mathrm{mg}$, a un ritmo de una por día.

Posteriormente, se realizaron pruebas de control durante los próximos 7 y 14 días del inicio del tratamiento, para comprobar el efecto de los productos utilizados. 


\section{RESULTADOS}

Tabla 1. Distribución porcentual de la infección vaginal por tipo y fecha

\begin{tabular}{|c|c|c|c|c|c|c|c|}
\hline \multirow{2}{*}{ TIPO } & & & \multicolumn{4}{|c|}{ INFECCIÓN VAGINAL } & \multirow{2}{*}{ 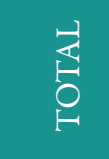 } \\
\hline & & & AUSENTE & ESCASO & MODERADO & $\begin{array}{l}\text { ABUNDANTE } \\
\text { AUSENTE }\end{array}$ & \\
\hline \multirow{3}{*}{ 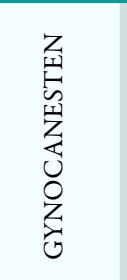 } & \multirow{3}{*}{ 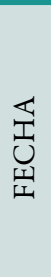 } & $\begin{array}{c}\text { FECHA } \\
17 / 05 / 2011\end{array}$ & & $44,4 \%$ & $44,4 \%$ & $11,1 \%$ & $100,0 \%$ \\
\hline & & $\begin{array}{c}\text { FECHA } \\
24 / 05 / 2011\end{array}$ & $33,3 \%$ & $66,7 \%$ & & & $100,0 \%$ \\
\hline & & $\begin{array}{c}\text { FECHA } \\
31 / 05 / 2011\end{array}$ & $44,4 \%$ & $55,6 \%$ & & & $100,0 \%$ \\
\hline Total & & $25.9 \%$ & $55,6 \%$ & $14,8 \%$ & $3,7 \%$ & $100,0 \%$ & \\
\hline \multirow{4}{*}{ 岁 } & \multirow{4}{*}{ 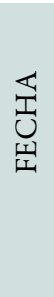 } & $\begin{array}{c}\text { FECHA } \\
17 / 05 / 2011\end{array}$ & & $44,4 \%$ & $44,4 \%$ & $11,1 \%$ & $100,0 \%$ \\
\hline & & $\begin{array}{c}\text { FECHA } \\
24 / 05 / 2011\end{array}$ & $33,3 \%$ & $66,7 \%$ & & & $100,0 \%$ \\
\hline & & $\begin{array}{c}\text { FECHA } \\
31 / 05 / 2011\end{array}$ & $22,2 \%$ & $77,8 \%$ & & & $100,0 \%$ \\
\hline & & Total & $18,5 \%$ & $63,0 \%$ & $14,8 \%$ & $3,7 \%$ & $100,0 \%$ \\
\hline \multirow{4}{*}{ 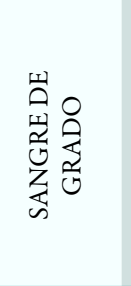 } & \multirow{4}{*}{ 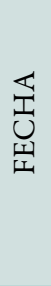 } & $\begin{array}{c}\text { FECHA } \\
17 / 05 / 2011\end{array}$ & & $44,4 \%$ & $44,4 \%$ & $11,1 \%$ & $100,0 \%$ \\
\hline & & $\begin{array}{c}\text { FECHA } \\
24 / 05 / 2011\end{array}$ & $22,2 \%$ & $77,8 \%$ & & & $100,0 \%$ \\
\hline & & $\begin{array}{c}\text { FECHA } \\
31 / 05 / 2011\end{array}$ & $11,1 \%$ & $88,9 \%$ & & & $100,0 \%$ \\
\hline & & Total & $11,1 \%$ & $70,4 \%$ & $14,8 \%$ & $3,7 \%$ & $100,0 \%$ \\
\hline
\end{tabular}

$\%$ de FECHA

Gráfico 1. Número de infecciones vaginales contrarrestadas por el Gynocanesten ${ }^{\circledR}$ entre el 17 y 31 de mayo

Tipo: Gynocanesten

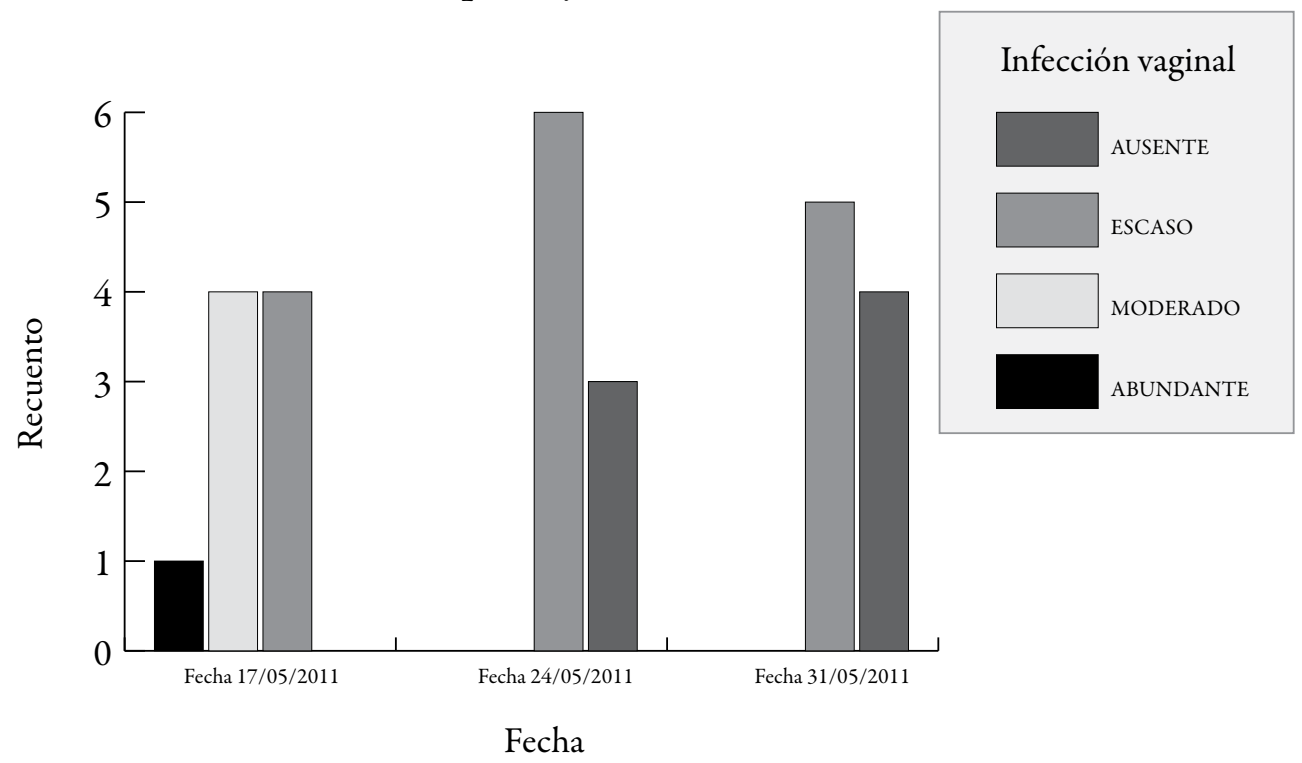


Gráfico 2. Número de infecciones vaginales contrarrestadas por el aloe vera entre el 17 y 31 de mayo

Tipo: Sabila

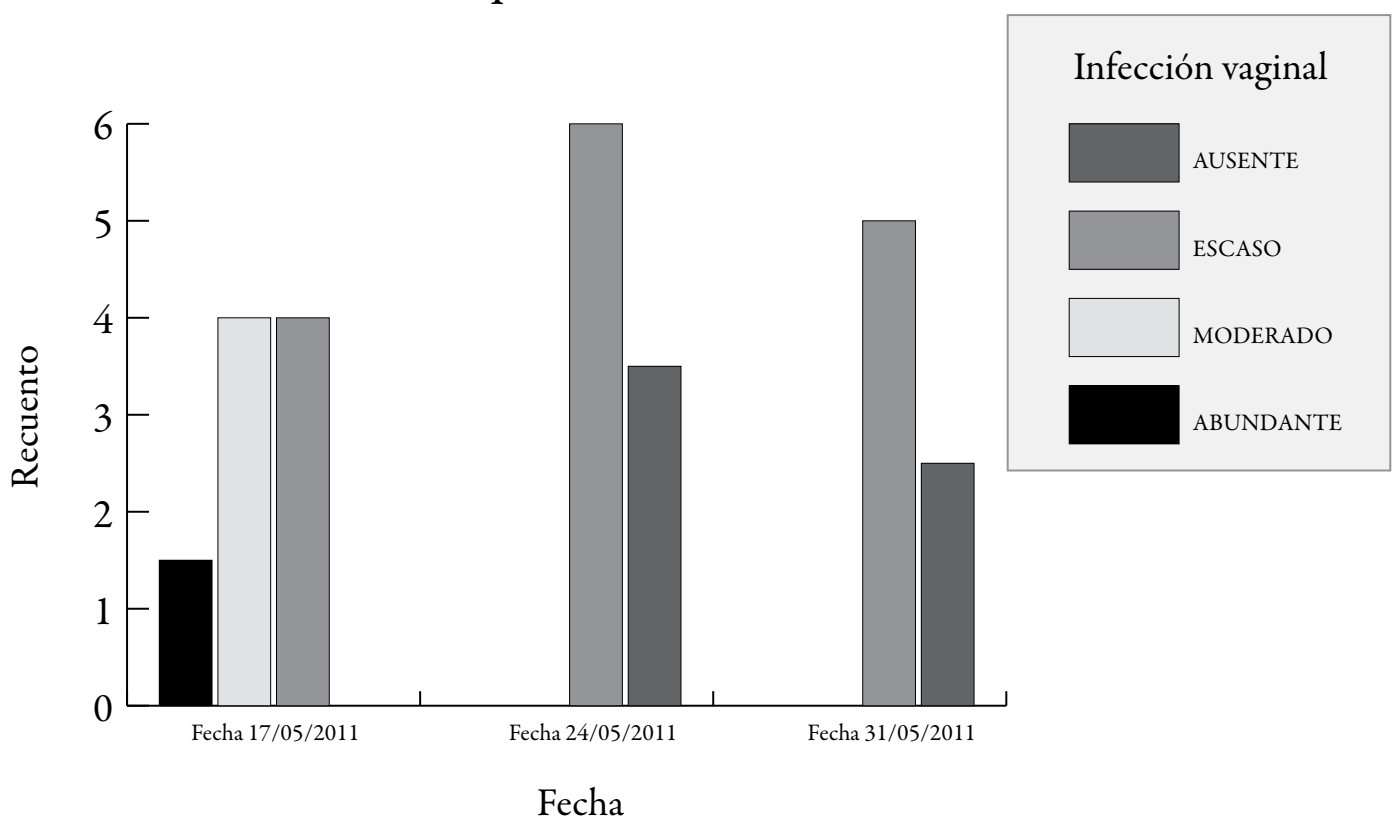

Gráfico 3. Número de infecciones vaginales contrarrestadas por la sangre de grado entre el 17 y 31 de mayo

Tipo: Sangre de grado

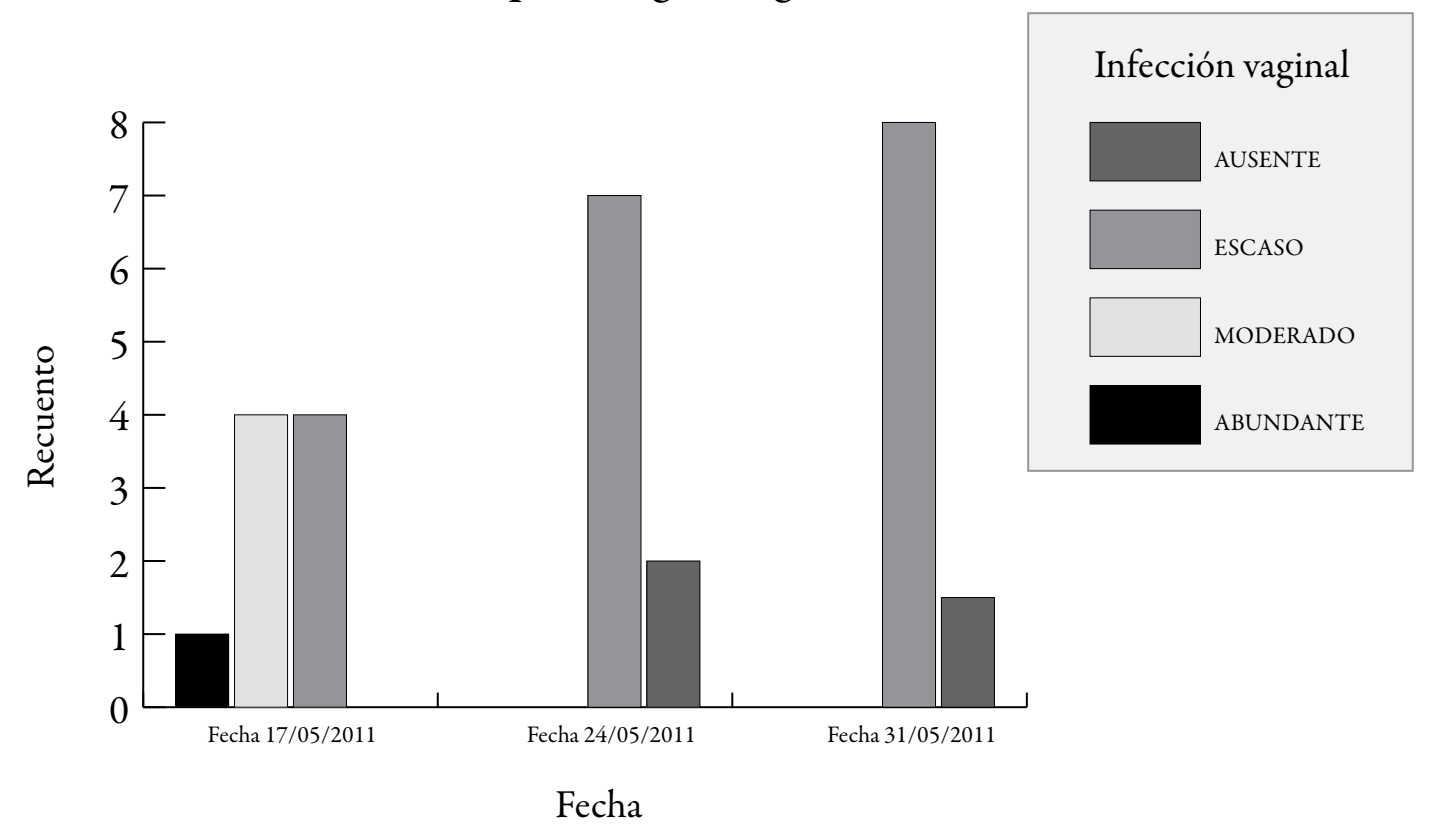


Eficacia antimicótica vaginal entre la sangre de grado y el Gynocanesten

La tabla ANOVA para los efectos nos prueba a un $95 \%$ de confianza que no existe diferencia significativa entre la aplicación de la sangre de grado y el Gynocanesten en la reducción de infecciones vaginales. Ambos productos llevan al mismo resultado en el tratamiento, lo que marca la eficacia de la sangre de grado frente al uso de Gynocanesten .

La prueba Duncan nos indica que existe una diferencia significativa entre los resultados de la fecha 17 de mayo frente a los del 24 y 31 de mayo respectivamente, donde se reducen significativamente las infecciones vaginales, como se puede verificar en las tablas siguientes:

Tabla 2. Factores intersujetos

\begin{tabular}{cccc} 
& & Etiqueta del valor & N \\
TIPO & 1 & GYNOCANESTEN $^{\circ}$ & 27 \\
& 3 & SANGRE DE GRADO & 27 \\
& 1 & FECHA 17/05/2011 & 18 \\
FECHA & 2 & FECHA 24/05/2011 & 18 \\
& 3 & FECHA 31/05/2011 & 18 \\
\hline
\end{tabular}

Tabla 3. Pruebas de los efectos intersujetos

Variable dependiente: INFECCIÓN VAGINAL

\begin{tabular}{|cccccc|}
\hline Fuente & Suma de cuadrados tipo III & gl & $\begin{array}{c}\text { Media } \\
\text { cuadrática }\end{array}$ & F & Significación \\
\hline $\begin{array}{c}\text { Modelo } \\
\text { corregido }\end{array}$ & $11,259(\mathrm{a})$ & 5 & 2,252 & 7,370 & 0,000 \\
\hline $\begin{array}{c}\text { Intersección } \\
\text { TIPO }\end{array}$ & 58,074 & 1 & 58,074 & 190,061 & 0,000 \\
\hline FECHA & 0,296 & 1 & 0,296 & 0,970 & 0,330 \\
\hline TIPO* & 10,704 & 2 & 5,352 & 17,515 & 0,000 \\
\hline FECHA & 0,259 & 2 & 0,130 & 0,424 & 0,657 \\
\hline Error & 14,667 & 48 & 0,306 & & \\
\hline Total & 84,000 & 54 & & & \\
\hline $\begin{array}{c}\text { Total } \\
\text { corregida }\end{array}$ & 25,926 & 53 & & & \\
\hline
\end{tabular}

a $\mathrm{R}$ cuadrado $=0,434(\mathrm{R}$ cuadrado corregida $=0.375)$ 


\section{Tabla 4. Infección vaginal \\ Duncan}

\begin{tabular}{|c|c|c|c|}
\hline & & \multicolumn{2}{|c|}{ Subconjunto } \\
\hline FECHA & $\mathrm{N}$ & $\begin{array}{l}1 \\
2\end{array}$ & 1 \\
\hline $\begin{array}{c}\text { FECHA } \\
24 / 05 / 2011\end{array}$ & 18 & 0,72 & \\
\hline $\begin{array}{c}\text { FECHA } \\
31 / 05 / 2011\end{array}$ & 18 & 0,72 & \\
\hline $\begin{array}{c}\text { FECHA } \\
17 / 05 / 2011\end{array}$ & 18 & & 1,67 \\
\hline Significación & & 1,000 & 1,000 \\
\hline
\end{tabular}

Se muestran las medias para los grupos en subconjuntos homogéneos.

Basado en la suma de cuadrados tipo III. El término error es la media cuadrática (Error) $=0,306$. a usa el tamaño muestral de la media armónica $=18,000$. Alfa $=0,05$.

\section{Gráfico 4. Medidas marginales estimadas de infección vaginal}
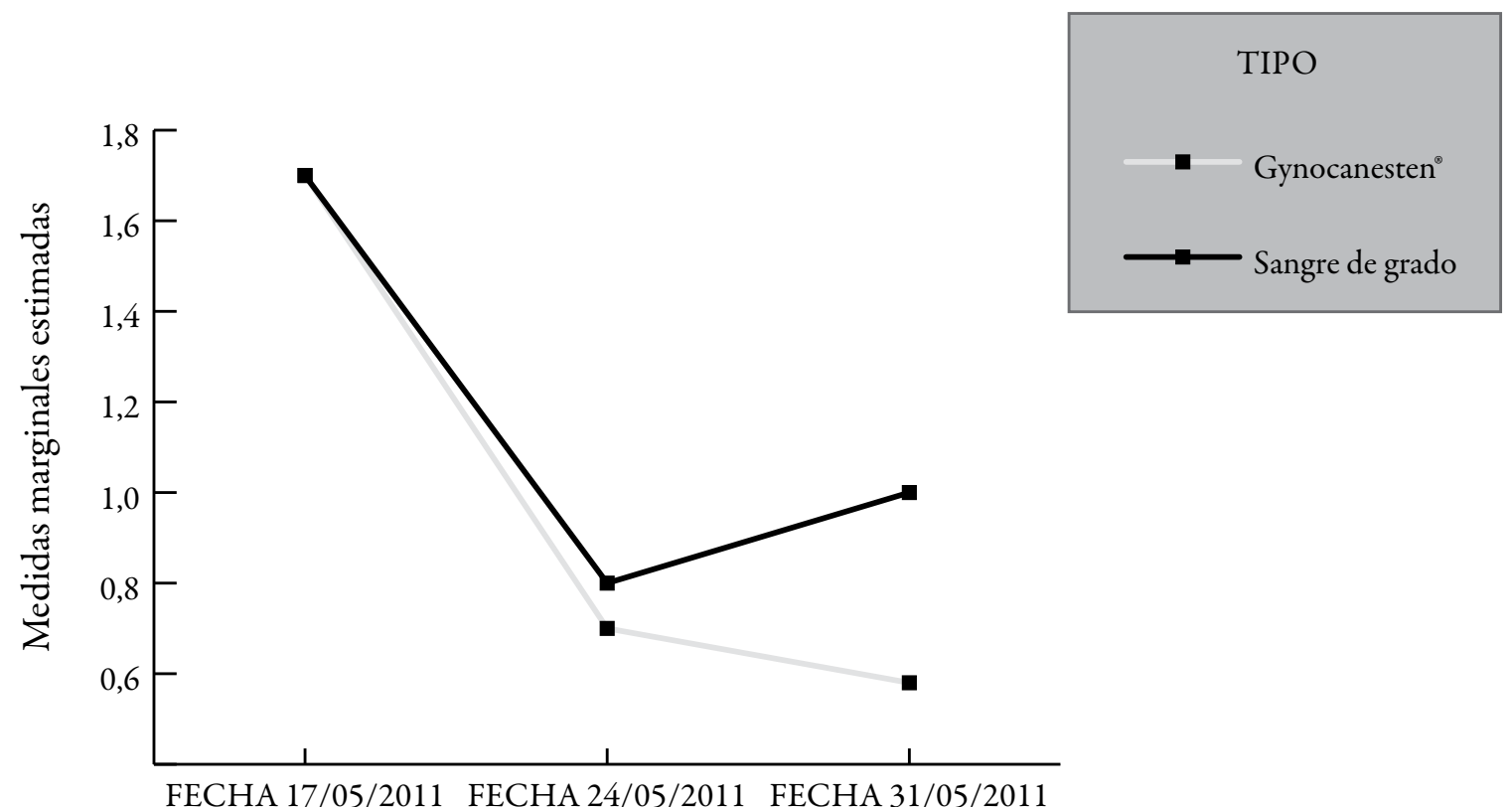

FECHA 
Eficacia antimicótica vaginal entre el aloe vera y el Gynocanesten ${ }^{\bullet}$

La tabla ANOVA nos indica para los efectos un $95 \%$ de confianza de que no existe diferencia significativa entre la aplicación del aloe vera y el Gynocanesten ${ }^{\circledR}$ en la reducción de infecciones vaginales. Ambos productos conducen al mismo resultado en el tratamiento, lo que marca la eficacia del aloe vera frente al uso de Gynocanesten .

La prueba Duncan nos indica que existe una diferencia significativa entre los resultados en la fecha del 17 de mayo frente a los del 24 y 31 de mayo respectivamente, donde se reducen significativamente las infecciones vaginales.

Tabla 5. Factores intersujetos

\begin{tabular}{cccc} 
& & Etiqueta del valor & N \\
\multirow{2}{*}{ TIPO } & 1 & GYNOCANESTEN $^{\circ}$ & 27 \\
& 2 & SÁBILA & 27 \\
& 1 & FECHA 17/05/2011 & 18 \\
FECHA & 2 & FECHA 24/05/2011 & 18 \\
& 3 & FECHA 31/05/2011 & 18 \\
\hline
\end{tabular}

Tabla 6. Pruebas de los efectos intersujetos

Variable dependiente: INFECCIÓN VAGINAL

\begin{tabular}{cccccc}
\hline Fuente & $\begin{array}{c}\text { Suma de } \\
\text { cuadrados } \\
\text { tipo III }\end{array}$ & gl & $\begin{array}{c}\text { Media } \\
\text { cuadrática }\end{array}$ & F & Significación \\
\hline $\begin{array}{c}\text { Modelo } \\
\text { corregido }\end{array}$ & $12,222(\mathrm{a})$ & 5 & 2,444 & 7,437 & 0,000 \\
\hline $\begin{array}{c}\text { Intersección } \\
\text { TIPO }\end{array}$ & 54,000 & 1 & 54,000 & 164,282 & 0,000 \\
\hline FECHA & 12,000 & 2 & 6,000 & 18,254 & 0,000 \\
\hline TIPO & 0,148 & 2 & 0,074 & 0,225 & 0,799 \\
FECHA & 15,778 & 48 & 0,329 & & \\
\hline Error & 82,000 & 54 & & & \\
\hline Total & 28,000 & 53 & & & \\
\hline Total corregida & & 1 & 0,074 & 0,225 & 0,637 \\
\hline
\end{tabular}

a $\mathrm{R}$ cuadrado $=0,437(\mathrm{R}$ cuadrado corregida $=0,378)$ 


\section{Tabla 7. Infección vaginal \\ Duncan}

\begin{tabular}{|cccc}
\hline & \multicolumn{3}{c}{ Subconjunto } \\
FECHA & N & 1 & 1 \\
& & 2 & \\
\hline FECHA 31/05/2011 & 18 & 0,67 & \\
\hline FECHA 24/05/2011 & 18 & 0,67 & \\
\hline FECHA 17/05/2011 & 18 & & 1,67 \\
\hline Significación & & 1,000 & 1,000 \\
\hline
\end{tabular}

Se muestran las medias para los grupos en subconjuntos homogéneos.

Basado en la suma de cuadrados tipo III. El término error es la Media cuadrática (Error) $=0,329$. a usa el tamaño muestral de la media armónica $=18,000$. Alfa $=0,05$.

\section{Gráfico 5. Medidas marginales estimadas de infección vaginal}

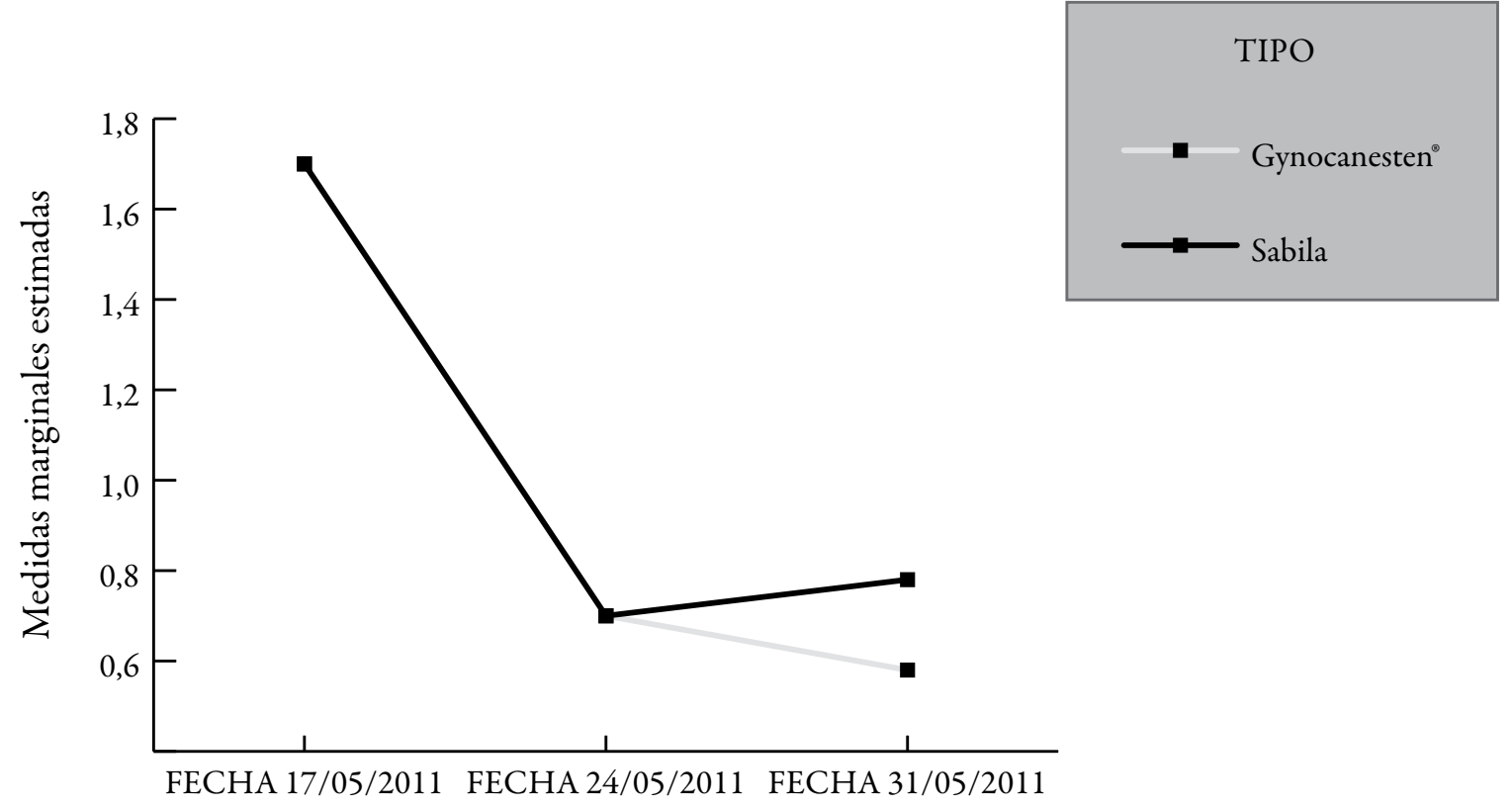

FECHA 
Eficacia antimicótica vaginal entre el aloe vera, sangre de grado y el Gynocanesten ${ }^{\bullet}$

Se contrasto la hipótesis con un nivel de confianza del $95 \%$ para probar la eficiencia del aloe vera y la sangre de grado comparados con el Gynocanesten', donde se encontró que existe igualdad en los resultados para los tres tipos de productos evaluados, lo que significa que el aloe vera y la sangre de grado producen los mismos resultados que el Gynocanesten. Además, la prueba nos indica que sí existe diferencia significativa en las tres fechas que se recolectaron los datos, lo que quiere decir que las infecciones vaginales se redujeron significativamente a la última fecha en que se recolectó la información.

\section{Tabla 8. Factores intersujetos}

\begin{tabular}{cccc} 
& & Etiqueta del valor & N \\
\multirow{4}{*}{ TIPO } & 1 & GYNOCANESTEN & 27 \\
& 2 & SÁBILA & 27 \\
& 3 & SANGRE DE GRADO & 27 \\
FECHA & 1 & FECHA 17/05/2011 & 27 \\
& 2 & FECHA 24/05/2011 & 27 \\
& 3 & FECHA 31/05/2011 & 27 \\
\hline
\end{tabular}

Tabla 9. Pruebas de los efectos intersujetos Variable dependiente: INFECCIÓN VAGINAL

\begin{tabular}{cccccc}
\hline Fuente & $\begin{array}{c}\text { Suma de } \\
\text { cuadrados tipo III }\end{array}$ & gl & $\begin{array}{c}\text { Media } \\
\text { cuadrática }\end{array}$ & F & Significación \\
\hline $\begin{array}{c}\text { Modelo } \\
\text { corregido }\end{array}$ & $16,667(\mathrm{a})$ & 8 & 2,083 & 6,750 & 0,000 \\
\hline Intersección & 87,111 & 1 & 87,111 & 282,240 & 0,000 \\
\hline TIPO & 0,296 & 2 & 0,148 & 0,480 & 0,621 \\
\hline FECHA & 16,074 & 2 & 8,037 & 26,040 & 0,000 \\
\hline TIPO $*$ & 0,296 & 4 & 0,074 & 0,240 & 0,915 \\
FECHA & 22,222 & 72 & 0,309 & & \\
\hline Error & 126,000 & 81 & & & \\
\hline Total & 38,889 & 80 & & & \\
\hline Total corregida & & & & \\
\hline
\end{tabular}

a $\mathrm{R}$ cuadrado $=0,429(\mathrm{R}$ cuadrado corregida $=0,365)$

Para la siguiente hipótesis se utilizó una prueba $\mathrm{F}$ para un análisis de varianza con dos factores (tipo de producto: Ggynocanesten, sábila y sangre de grado, y tres fechas: 17, 24 y 31 de mayo del 2011). 


\section{PRUEBAS POST HOC FECHA}

\section{Tabla 10. Subconjuntos homogéneos}

INFECCIÓN VAGINAL

Duncan

\begin{tabular}{cccc} 
FECHA & N & \multicolumn{2}{c}{ Subconjunto } \\
& 1 & 2 & 1 \\
\hline FECHA 24/05/2011 & 27 & 0,70 & \\
\hline FECHA 31/05/2011 & 27 & 0,74 & \\
\hline FECHA 17/05/2011 & 27 & & 1,67 \\
\hline Significación & & 0,807 & 1,000 \\
\hline
\end{tabular}

Se muestran las medias para los grupos en subconjuntos homogéneos.

Basado en la suma de cuadrados tipo III. El término error es la media cuadrática (Error) $=0,309$.

a usa el tamaño muestral de la media armónica $=27,000$. Alfa $=0,05$.

La tabla anterior (Prueba de Límite de Significancia de Duncan) nos indica la diferencia que existe en los resultados de infecciones vaginales de la primera fecha (17 de mayo del 2011) con respecto a las otras dos fechas (24 y 31 de mayo del 2011). Esto quiere decir que los tres productos reducen significativamente las infecciones vaginales, con lo que se prueba la eficiencia del aloe vera y la sangre de grado con respecto a la aplicación de Gynocanesten.

\section{Gráfico 6. Medidas marginales estimadas de infección vaginal}

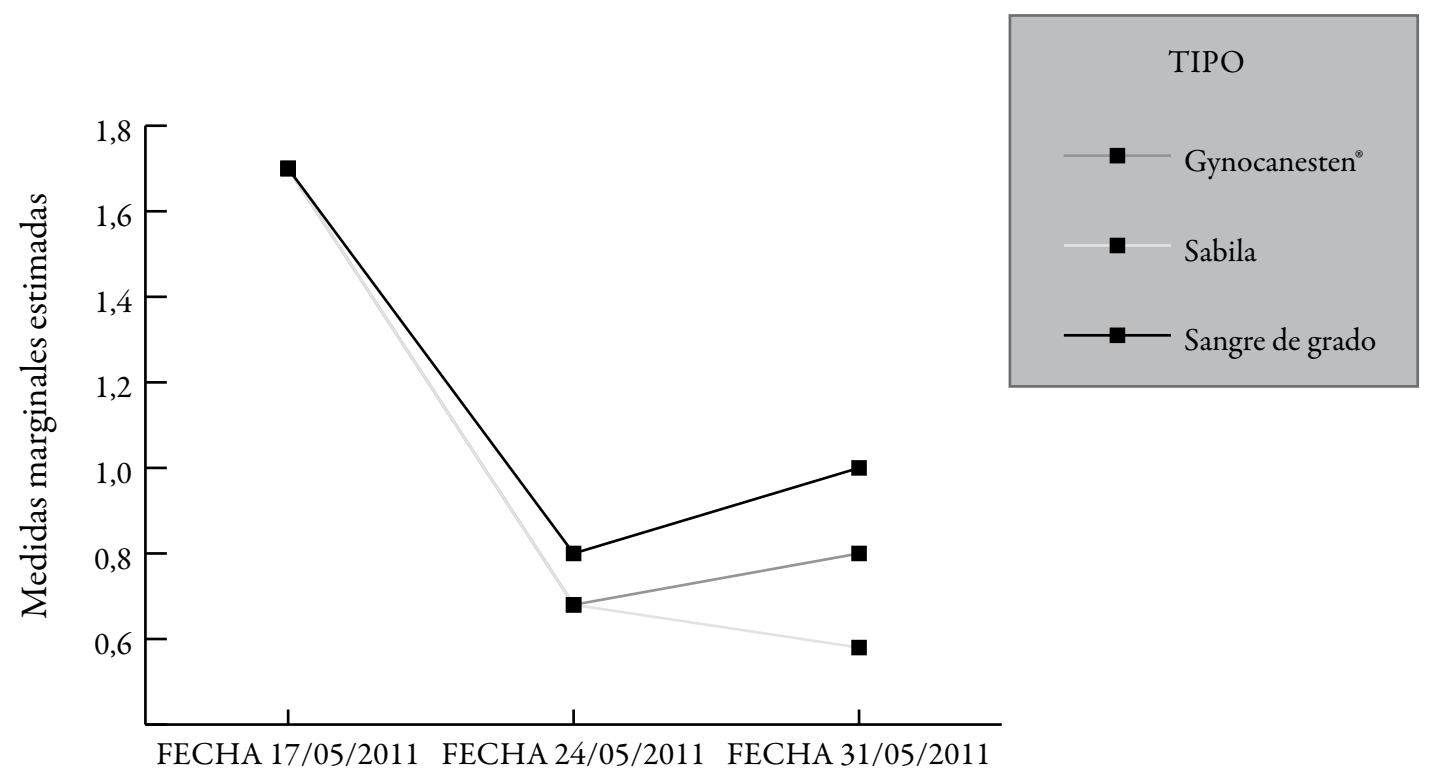

FECHA 


\section{CONCLUSIONES}

Para analizar los resultados se empleó la tabla ANOVA, la cual arrojo que al comparar la sangre de grado con el producto control Gynocanesten', así como también el aloe vera con el producto control, no hubo mayor diferencia al emplear uno u otro de ellos en sus grupos respectivos durante el tratamiento de las infecciones vaginales. Sin embargo, al realizar la prueba de Duncan se pudo observar una variación durante los días 24 y 31 posteriores al tratamiento, pero aun así, no perdió la eficacia de los productos en evaluación. Por lo tanto, se concluyó que existe eficacia antimicótica vaginal entre la sangre de grado y el Gynocanesten, igual que existe eficacia antimicótica vaginal entre el aloe vera y el Gynocanesten.

Finalmente, al realizar el estudio comparativo de los tres productos, se muestra que hubo una diferencia significativa de la evolución de la infección vaginal durante el tratamiento, percibiéndose esta diferencia de forma más evidente durante las últimas fechas del estudio, al aplicar la Prueba de Límite de Significancia de Duncan. Por ello, con estos resultados se pudo concluir que existe eficacia antimicótica vaginal por parte del aloe vera y la sangre de grado al aplicarlos al tracto vaginal en el transcurso de dos semanas mientras, que el Gynocanesten logra el mismo efecto en el tracto vaginal en solo seis, días y se conserva el efecto del mismo al evaluarlo a las 2 semanas de iniciado el tratamiento, a pesar de que se dejó de usar a los seis días.

\section{DISCUSIÓN}

De acuerdo con varias publicaciones hechas en diversas revistas científicas citadas anteriormente, se está al tanto de que las infecciones vaginales son muy frecuentes en las mujeres en edad fértil, las mismas que muchas veces son asintomáticas y no tratadas por los altos costos de los tratamientos farmacológicos.
Sin embargo, durante años, muchos estudios fitoterápicos nos han dado a conocer las propiedades de diversos productos naturales, resaltando sus características con evidencia científica.

$\mathrm{Al}$ realizar nuestro estudio científico sobre la eficacia del aloe vera y la sangre de grado en las infecciones vaginales, este ha podido ser avalado por otros investigadores como Ortiz, Capcha, Palomino, Aguilar (2003). Además, debemos considerar las propiedades antimicóticas y antivíricas que presenta la sangre de grado, aunque en este caso nos hemos referido básicamente a la actividad antibacteriana de la sangre de grado frente a la bacteria. Los resultados obtenidos muestran que la sangre de grado inhibe el crecimiento de Helicobacter pylori en concentraciones elevadas, y también se determinó el efecto bactericida del producto concentrado. Esta investigación ha logrado establecer también resultados positivos en el tratamiento de las infecciones vaginales causados por bacterias y hongos.

Además, con respecto a los estudios sobre el aloe vera, Bland (1985) nos reporta que el beber gel de aloe vera mejora la ingestión de las proteínas, ayuda a normalizar el movimiento intestinal y controla las infecciones de hongos, además de mejorar el síndrome del colon irritable, la acidez estomacal y la colitis. Se ha demostrado, de esta manera, que el tratamiento con aloe vera controla e inhibe el crecimiento micótico, hecho comprobado en nuestro estudio al controlar y reducir la infección vaginal.

Teniendo evidencia científica vigente sobre las propiedades antibacterianas y antimicóticas del aloe vera y la sangre de grado, se demuestra la gran eficacia que poseen si se aplica de forma adecuada en el tracto vaginal con presencia de hongos. 


\section{REFERENCIAS BIBLIOGRÁFICAS}

1. Baudo G. "Aloe vera". ErboristeriaDomani. 1992; 2: 29-33

2. Bland J. Del Instituto Linus Pauling reportó que el beber gel de aloe vera mejora la ingestión. 1985.

3. Hay P. National guideline for the management of bacterial vaginosis. Clinical Effectiveness Group. British Association for Sexual Health and HIV, 2006.

4. Méndez M., Calderón J., Soria A., Yui M., Apaza N. "Vaginosis bacteriana: diagnóstico y prevalencia en un centro de salud". Revista Peruana de Ginecología y Obstetricia. Vol. 47, No1, enero del 2001.

5. Ortiz Tamariz, Humberto J.; Capcha Mendoza, Roberto; Palomino Cadenas, Edwin J.; Aguilar Olano, José. "Actividad antibacteriana de la sangre de grado (Crotonlechleri) frente al Helicobacter pylori. Revista Medicina Herediana, v. 14, № 2, Lima, abr. 2003. ISSN 1018-130X. Disponible en www.upch. edu.pe/famed/rmh/14-2/v14n2ao6.pdf

6. Spence D., Mellville C. "Vaginal discharge". BMJ 2007; 335: 1147-51. 